\title{
Occult Cerebral Aspergillosis Presenting with Focal Status Epilepticus in an Immunocompetent Patient
}

\author{
Anil Ramineni*, Sarah Zubkov, Madeline Fields and Lara Marcuse
}

Mount Sinai Hospital, Neurology, New York, USA

Received: March 18, 2015; Accepted: June 29, 2015; Published: July 02, 2015

*Corresponding author: Anil Ramineni, Mount Sinai Hospital, Neurology, New York, USA, E-mail: anil.ramineni@mssm.edu

\begin{abstract}
Invasive aspergillosis is classically viewed as a disease of the immunocompromised patient. We describe the case of an immunocompetent neurosurgical patient who presented with occult cerebral aspergillosis manifesting as focal status epilepticus. We highlight the diagnostic dilemmas in managing this case, and review guidelines for treatment of cerebral aspergillosis.
\end{abstract}

\section{Case Report}

SC, a 64-year-old man, sustained a moderate traumatic brain injury resulting in a depressed left parietotemporal skull fracture which was treated neurosurgically via cranioplasty. Post-operatively, he received one week of seizure prophylaxis with phenytoin and then was discharged without event. He had minimal residual neurological deficits aside from occasional paraphasic errors in language.

Eight months later, he abruptly became lethargic with right face and arm rhythmic, jerking movements. He was admitted to a community hospital and diagnosed with focal seizures after a routine EEG showed left hemispheric spikes as well as two electrographic seizures affecting the left hemisphere. He was treated with levetiracetam as well as valproic acid. The patient was afebrile, his laboratory studies including HIV were normal, and blood cultures had no growth. His jerking movements resolved, however he remained lethargic. An MRI scan of his head without contrast demonstrated evidence of post-operative changes near his cranioplasty site, but no acute abnormality.

He was transferred to our institution after 1 week for further medical evaluation. On arrival, he was minimally arousable to noxious stimulation, non-verbal, moving all extremities with hyperreflexia and a Babinski sign present on the right side. He was placed on continuous EEG monitoring which revealed complex periodic discharges involving the left parietotemporal region concerning for nonconvulsive status epilepticus. Phenytoin and oxcarbazepine were added to his anti-epileptic regimen, but his mental status remained unchanged.

An MRI with contrast exhibited a 9mm ring enhancing left parietal lesion with surrounding vasogenic edema (Figure 1) that was not apparent on prior imaging without contrast. Infectious disease was consulted and recommended broadspectrum empiric antibacterial therapy due to suspicion of an abscess. Neurosurgery was consulted and the patient was taken to the OR where a new posterior cranioplasty was performed. The bone appeared grossly infected and the specimen was sent for culture. Despite successful surgery, his poor mental status persisted. Initial operative cultures were unrevealing, and he was continued on anti-bacterial treatment. Three days later, surgical pathology returned with numerous fungal organisms. His antibiotic regimen was broadened to include voriconazole. Over the next several days, he became more alert and talkative and his anti-epileptic regimen was tapered and antibacterial therapy was
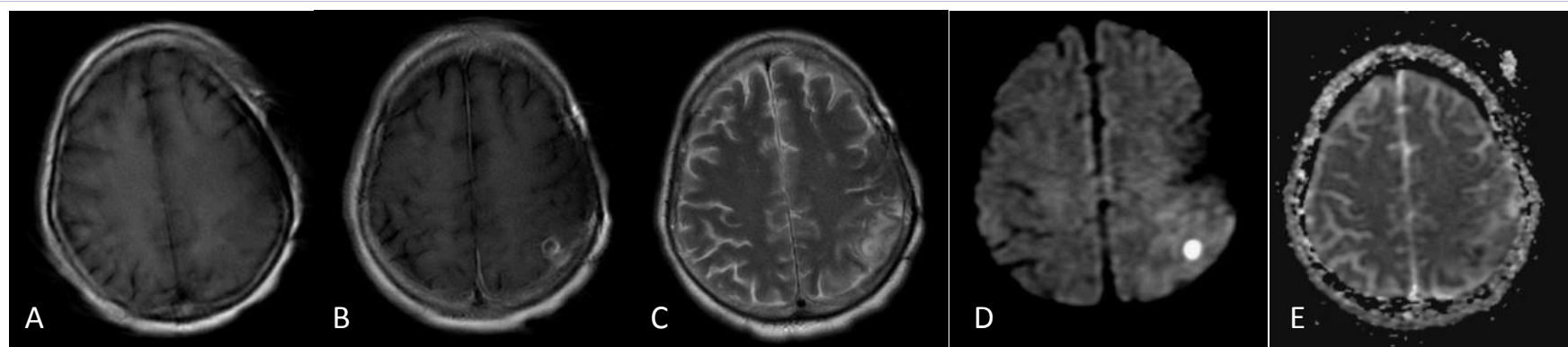

Figure 1: MRI brain with and without contrast.

(A) left parietal lobe lesion is shown as a hypointense focus on T1 sequence, (B) a ring-enhancing lesion with administration of contrast, (C) a hyperintense lesion with surrounding vasogenic edema on T2 sequence, (D) with restricted diffusion, (E) and true ADC dropout. 
discontinued. Fungal speciation revealed aspergillus fumigatus. Upon discharge, the patient had returned to his prior neurologic baseline with the exception of mild deconditioning and he was prescribed a prolonged course of voriconazole with infectious disease follow-up.

\section{Discussion}

Aspergillus is a ubiquitous soil-dwelling organism found in water, dust, compost, foods, spices, unfiltered air, and plants [1]. Despite the fact that people inhale numerous spores on a daily basis, it rarely manifests pathologically in the immunologically competent individual. The majority of literature focuses on classic risk factors which predispose individuals to invasive aspergillus infections. These include severe neutropenia, high dose glucocorticoids or other immunosuppressants, and HIV/ AIDS $[2,3,4]$. The majority of infections are attributable to Aspergillus fumigatus (with Aspergillus flavus and Aspergillus niger contributing in a minority of cases) [4].

Aspergillus species grow well on standard media and can be identified to species level in most laboratories. Culture confirmation is important to differentiate aspergillosis from other filamentous fungal infections. Blood cultures are of limited utility as they are often not positive even in disseminated infection [5]

A variety of post-operative complications of Aspergillus have been described including endocarditis, aortitis, and mediastinitis following open-heart surgery. Additionally, aspergillosis following breast, orthopedic, and ophthalmologic surgery has also been described [6-11].

Cerebral aspergillosis as a late complication of neurosurgical intervention is often forgotten [12]. Our patient had an unusual presentation with focal status epilepticus that failed to improve despite numerous anti-epileptic medications. MRI imaging with gadolinium proved essential in making the diagnosis. Our suspicion for an underlying lesion was high, despite the absence of fever, due to the focal nature of his seizures. Ultimately, the patient required neurosurgical re-exploration as well as prolonged antifungal treatment and close follow-up to ensure eradication of his infection. Voriconazole compares favorably to amphotericin B in management of CNS aspergillosis and achieves excellent CSF penetration [5]. While voriconazole is less nephrotoxic, care must be taken to monitor liver function tests. Optimal duration of treatment is unclear, although most authorities recommend continuing antifungals until clinical and radiologic improvement and a minimum of 6-12 weeks [13]. Our patient received a 10 week course of antifungal treatment with resolution in radiologic disease and no further symptoms.

\section{References}

1. Soubani AO, Chandrasekar PH. The clinical spectrum of pulmonary aspergillosis. Chest. 2002;121:12065367.

2. Lewis RE, Kontoyiannis DP. Invasive aspergillosis in glucocorticoidtreated patients. Med Mycol. 2009;47 Suppl 1:18654923. doi:10.1080/13693780802227159.

3. Lilic D. Unravelling fungal immunity through primary immune deficiencies. Curr Opin Microbiol. 2012;15:22818901. doi:10.1016/j. mib.2012.06.003.

4. Mylonakis E, Barlam TF, Flanigan T, Rich JD. Pulmonary aspergillosis and invasive disease in AIDS: review of 342 cases. Chest. 1998;114:9674477.

5. Schwartz S, Ruhnke M, Ribaud P, Corey L, Driscoll T, Cornely OA, et al. Improved outcome in central nervous system aspergillosis, using voriconazole treatment. Blood. 2005;106:15998833. doi:10.1182/ blood-2005-02-0733.

6. Ellis ME, Al-Abdely H, Sandridge A, Greer W, Ventura W. Fungal endocarditis: evidence in the world literature, 1965-1995. Clin Infect Dis. 2001;32:11118386. doi:10.1086/317550.

7. Sanchez-Recalde A, Maté I, Merino JL, Simon RS, Sobrino JA. Aspergillus aortitis after cardiac surgery. J Am Coll Cardiol. 2003;41:12570958.

8. Dimopoulos G, Tsangaris I, Poulakou G, Panayiotides J, Tsaknis G, Orfanos S, et al. Post-operative Aspergillus mediastinitis in a man who was immunocompetent: a case report. J Med Case Rep. 2010;4:20863378. doi:10.1186/1752-1947-4-312.

9. Williams K, Walton RL, Bunkis J. Aspergillus colonization associated with bilateral silicone mammary implants. Plast Reconstr Surg. 1983;71:6823489.

10. Peters-Christodoulou MN, de Beer FC, Bots GT, Ottenhoff TM, Thompson J, van 't Wout JW. Treatment of postoperative Aspergillus fumigatus spondylodiscitis with itraconazole. Scand J Infect Dis. 1991;23:1652789.

11. Mendicute J, Orbegozo J, Ruiz M, Sáiz A, Eder F, Aramberri J. Keratomycosis after cataract surgery. J Cataract Refract Surg. 2000;26:11084276.

12. Bokhari R, Baeesa S, Al-Maghrabi J, Madani T. Isolated cerebral aspergillosis in immunocompetent patients. World Neurosurg. 2014;82:24076053. doi:10.1016/j.wneu.2013.09.037.

13. Walsh TJ, Anaissie EJ, Denning DW, Herbrecht R, Kontoyiannis DP, Marr KA, et al. Treatment of aspergillosis: clinical practice guidelines of the Infectious Diseases Society of America. Clin Infect Dis. 2008;46:18177225. doi:10.1086/525258. 